\title{
Miguel de Cervantes en The Monthly Review y The Critical Review: la prosa $(1749-1800)^{1}$
}

Recibido: 16/5/2017

Aceptado: 7/11/2017

\section{RESUMEN:}

Cervantes goza de un enorme protagonismo en la literatura británica del siglo XVIII. Su influencia se pone de manifiesto en Joseph Andrews, que Henry Fielding subtituló "Written in Imitation of the Manner of Cervantes", y en producciones de autores como Tobias Smollett o Charlotte Lennox. La visión de la crítica cobra un valor significativo a mediados de siglo, con el nacimiento de The Monthly Review y The Critical Review que proporcionaban exámenes de novedades bibliográficas. En este trabajo se plantea el estudio de la huella cervantina en la novela británica a partir de las valoraciones críticas producidas por ambas publicaciones periódicas desde sus inicios hasta finales de siglo.

PALABRAS CLAVE: Cervantes, publicaciones literarias, Gran Bretaña, novela, siglo XVIII

Miguel de Cervantes in The Monthly Review and The Critical Review: the prose (1749-1800)

\section{ABSTRACT:}

Cervantes is seen as an important figure in eighteenth-century British literature. His influence is evident in Joseph Andrews, which was subtitled "Written in Imita-

1 Este trabajo forma parte del proyecto «Recepción e interpretación del Quijote (1605-1830). Traducciones, opiniones, recreaciones» (Ministerio de Economía e Innovación, ref.: FFI2014-56414-P) 
tion of the Manner of Cervantes", and in other works, most notably those by Tobias Smollett and Charlotte Lennox. From the middle of the century, the appearance of The Monthly Review and The Critical Review, which published evaluations of newly printed works, emphasized the importance of review criticism. In order to contribute to the study of the reception of Cervantes in the British novel, this paper examines book reviews produced by both periodical publications from their initial circulation until the end of the century.

KEYWORDS: Cervantes, Reviews, Great Britain, novel, eighteenth century.

Las publicaciones periódicas fueron una característica de la vida cultural británica del siglo dieciocho. Su estrecha vinculación con el mundo de la literatura se atestigua en la producción de documentos impresos por parte de prestigiosos referentes del panorama literario de aquella época. No fueron pocos los autores de renombre que proyectaron su experiencia a la prensa periódica e hicieron llegar su pensamiento ideológico y cultural a la ciudadanía. Daniel Defoe (c.1660-1731), Joseph Addison (16721719), Richard Steele (1672-1729), Jonathan Swift (1667-1745), Samuel Richardson (1689-1761), Henry Fielding (1707-1754), Samuel Johnson (1709-1784) y Tobias Smollett (1721-1771) son algunos de los escritores que participaron activamente en este proceso de desarrollo cultural.

A comienzos de siglo, concretamente en el año 1704, Defoe publicaba The Review (1704-1713). Unos años más tarde, Steele sacaba a la luz The Tatler (1709-1711), a la que muy pronto se sumaría Addison y, en 1710 nacía The Examiner (1710-1714) que contó con la colaboración de Swift. En 1711, Steele y Addison se embarcaron en una nueva y ambiciosa iniciativa editorial que no era otra que la fundación de la publicación The Spectator (1711-1712) (Cranfield, 1978: 35-36). La carrera de autores como Richardson y Fielding se caracteriza también por el apoyo de gestión y literario a la prensa. Richardson fue uno de los propietarios de The Daily Gazetteer (1735-1745) (Haig, 1960: 19). Fielding se vinculó a diversos proyectos periodísticos a finales de la década de los treinta y de los cuarenta en las publicaciones The Champion 
(1739-1742), que incluía sus escritos con el seudónimo Captain Vinegar (Marr, 1971: 108-109), The True Patriot (1745-1746) y The Jacobite's Journal (1747-1748) (Godden, 2008: 71-72, 97-101). Ya en 1752, fundaba The Convent-Garden (1752), en la que empleaba el seudónimo de Sir Alexander Drawcansir, Knt. Censor of Great Britain (Godden, 2008: 150). Samuel Johnson sacaba a la luz The Rambler (1750-1752), en la que se daba cobertura a temas relacionados con la literatura (Cranfield, 1978: 54). La participación de estos escritores en el mundo periodístico resalta su papel en el desarrollo de la cultura social de aquella sociedad.

En esta época ilustrada se imprimían publicaciones especializadas que mantenían informados a los lectores sobre el avance permanente del conocimiento en una amplia variedad de campos del saber. Michael de la Roche desarrolló una intensa actividad periodística que se tradujo en la edición de revistas literarias como Memoirs of Literature (1710-1714; 1717), New Memoirs of Literature (1725-1727) y A Literary Journal (1730-1731). En sus páginas se incluían artículos sobre trabajos que habían sido publicados recientemente. En aquella época aparecía también The Present State of the Republick of Letters (1728-1736), de Andrew Reid, que contenía informes sobre producciones que habían visto la luz tanto en Gran Bretaña como en el extranjero. Su objetivo, tal y como se indica en la página del título, era ofrecer una visión general del estado del conocimiento en Europa así como dar difusión a los libros más valiosos recién impresos en Gran Bretaña y en otros lugares del extranjero mediante artículos y resúmenes imparciales y precisos (The History of the Works of the Learned 1, enero-junio 1737). En 1736 esta cabecera se fusionó con The Literary Magazine (1735-1736) y pasó a denominarse The History of the Works of the Learned (1737-1742) (Graham, 1930: 201). Tras su desaparición en 1742, su lugar fue ocupado por The Literary Journal, una revista dublinense que se publicó entre los años 1744 y 1749 (Griffin, 2014: 127).

En 1749 el librero Ralph Griffiths (1720-1803) fundaba The Monthly Review (1749-1845) que influyó de manera notable en la 
historia de la crítica literaria británica. En primer lugar porque, a diferencia de las publicaciones que habían visto la luz con anterioridad, ofrecía comentarios críticos de trabajos enmarcados dentro de la poesía, la novela y el teatro y, en segundo lugar, porque proporcionaba observaciones así como resúmenes y citas de las obras reseñadas (Roper, 1978: 20). La identificación de todos los trabajos que habían salido recientemente a la luz así como la entrega de evaluaciones regulares de los mismos representó una ampliación de los formatos de revistas anteriores, tales como $\mathrm{Me}$ moirs of Literature and The History of the Works of the Learned (Keeran y Bowers, 2013: 163). Estas características novedosas convierten a The Monthly Review en una cabecera de prensa de interés para el estudio de la recepción de Cervantes en la prosa británica de la segunda mitad del siglo XVIII así como los textos publicados en The Critical Review (1756-1817). Al igual que la anterior, esta fuente hemerográfica ofrecía valoraciones de todas y cada una de las obras publicadas en aquel momento. Fundada por el impresor Archibald Hamilton (1719-1793) junto con el escritor Tobias Smollett, que asumió el papel de editor, The Critical Review también incluía un nuevo debate y método crítico que proporcionaba un contexto propicio para el desarrollo de la crítica literaria. Ciertamente, el afán de divulgación bibliográfica de The Monthly Review y The Critical Review, que permitía a los lectores obtener un acercamiento a obras muy diversas tanto británicas como extranjeras, resultó beneficioso para generar reflexiones en torno a textos producidos por un buen número de escritores y favoreció, a su vez, la construcción de un pensamiento y discurso crítico literario en la sociedad de la época. Estas orientaciones valorativas fueron influencias que probablemente contribuyeron a definir la posición literaria del lector, dado que le permitían enfocar las obras desde una perspectiva más informada y analíti$\mathrm{ca}$, al tiempo que determinaron el contenido de las bibliotecas y afectaron a la formación del canon (Basker, 2005: 329).

Desde este enfoque metodológico, y con el propósito de abrir nuevas aportaciones al estudio de la huella cervantina en la no- 
vela británica del siglo XVIII a partir de los comentarios críticos de la prensa literaria, se ha realizado una búsqueda y recopilación de artículos generados por The Monthly Review y The Critical Review. El análisis de estos documentos permite realizar un estudio comparado del contenido informativo de ambas revistas en lo relativo exclusivamente a la recepción de Cervantes y dilucidar el modo en que cada una de ellas difundió a la audiencia su influencia en la prosa británica en el periodo de tiempo que abarca desde 1749 hasta $1800 .^{2}$

La literatura española y el mérito de algunos de sus escritores han quedado registrados en los artículos de estas dos publicaciones periódicas. Los contenidos de algunas de sus reseñas pronto demuestran las notables dimensiones que el influjo de Cervantes había adquirido en la producción de autores como Henry Fielding, Charlotte Lennox (1730?-1804), Tobias Smollett o Richard Graves (1715-1804), hasta el punto de ser testimonio de la profunda huella que su apreciación del creador del Quijote dejó en la literatura moderna británica, en la que se evidencia, además,

2 La publicación esporádica de otras fuentes de similar orientación limita la posibilidad de evaluar la recepción de Cervantes a lo largo de todo el periodo objeto de estudio de manera completa. Pese a ello, sería pertinente analizar estas fuentes en futuros trabajos con el fin de contrastar los resultados obtenidos del conjunto de las cabeceras literarias y ofrecer una visión más amplia sobre la historia crítica de la recepción de Cervantes y su obra en Gran Bretaña en la segunda mitad del siglo XVIII. Entre estas cabeceras cabe hacer referencia a The General Review (1752), The Literary Magazine; or Universal Review (1756-1758), The Weekly Magazine and Literary Review (1758), The Theatrical Review (1763), The Edinburgh Magazine and Review (17731776), The London Review of English and Foreign Literature (1775-1780), The New Review (1782-1786), The English Review (1783-1796), The General Magazine and Impartial Review (1787-1792), The Analytical Review (1788-1799), The British Critic (1793-1826) y The Anti-Jacobin Review (1798-1810). Asimismo, investigaciones complementarias en publicaciones que circulaban en siglos posteriores, tales como The Edinburgh Review (1803-1929), The Saturday Review of Politics, Literature Science and Art (1855-1931), The London Mercury (1919-1939), The Criterion (1922-1939) o The National Review (18831950), posibilitaría, además, estudiar la evolución de los juicios críticos que se formularon relativos a este autor y ofrecer un examen más exhaustivo de esta temática en un periodo de tiempo más extenso. 
la dimensión internacional del insigne autor. ${ }^{3}$ No obstante, se debe señalar que estos no son todos los escritores de prosa que se conozca que estuvieron influenciados por Cervantes debido a la práctica existente en aquel momento de publicar de manera anónima (Raven, 2003: 143). Este modelo de escritura también se trasladó a las reseñas, lo cual imposibilita atribuir la autoría a estos textos. Ambas cabeceras se vendían por suscripción a lectores pertenecientes a las clases media y alta así como a instituciones educativas, sociedades literarias y bibliotecas. Esta tendencia asienta algunas argumentaciones para pensar que estas fuentes tenían buenas razones para no ofrecer artículos sesgados, falseados o interesados (Roper, 1978: 24-25). Oponerse o sobreproteger a un determinado autor podía hacer quedar en entredicho a la publicación, poner en tela de juicio su prestigio y afectar de forma notoria a su circulación. Tal y como establece Roger Roper, articular una crítica sesgada significaría poner en riesgo la repu-

3 La recepción de Cervantes en la literatura británica del siglo XVIII ha sido estudiada en diversas obras colectivas, tales como The Cervantean Heritage. Reception and Influence of Cervantes in Britain (2009), La huella de Cervantes y del Quijote en la cultura anglosajona (2007) y Cervantes in the English Speaking World (2005). A título individual, cabe hacer referencia a los estudios de Bautista Naranjo, La recepción y rescritura del mito de Don Quijote en Inglaterra (siglos XVII-XIX) (2015), Borham Puyal, Quijotes con enaguas. Encrucijada de géneros en el siglo XVIII británico (2015), Müllenbrock, «Don Quijote and Eighteenth-Century English Literature» (1999), Paulson, Don Quixote in England. The Aesthetics of Laughter (1998), Ballesteros, «La presencia de Don Quijote de Cervantes en Joseph Andrews de Fielding» (1989), Acosta Aide, «El influjo del El Quijote en Joseph Andrews» (1985) y Pardo García, «El Siglo de Oro del Quijote en la literatura inglesa, 1740-1840» (2007), «El Quijote y la novela inglesa: de Laurence Sterne a James Joyce» (2005), La tradición cervantina en la novela inglesa del siglo XVIII (1997a) y «Formas de imitación del Quijote en la novela inglesa del siglo XVIII: Joseph Andrews y Tristam Shandy» (1997b). Garrido Ardila también ha contribuido a la investigación de la impronta cervantina en la novela inglesa con «Las rutas del Quijote por la novela inglesa del siglo XVIII» (2016), Cervantes en Inglaterra: el Quijote y la novela inglesa del siglo XVIII (2014), «Un ejemplo señero de la Cervantean fiction: Roderick Random de Tobias Smollett» (2006), «La teoría cervantina de la novela en Roderick Random» (2002), Cervantes y la Quixotic Fiction: sucesión episódica y otros recursos narrativos (2001a), «Cervantes y la Quixotic Fiction: el hibridismo genérico» (2001b) y «Cervantes y la Quixotic Fiction: la parodia de géneros» (1998). 
tación de la revista (1978: 34). ${ }^{4}$ No obstante, esto no descartaba la emisión de juicios de valor sobre las obras reseñadas a pesar de que, en aquel momento, las citas tenían un lugar más destacado en estos textos. Además de ofrecer resúmenes y extractos de los trabajos, en ellos también se proporcionaba el título, nombre del autor, si se conocía, tamaño, precio y el nombre del editor, así como la opinión del evaluador sobre sus méritos o defectos.

Ya en la primera mitad de siglo, los trabajos de Henry Fielding Don Quixote in England, una obra teatral representada en los años treinta, en la que se traslada al caballero andante y a su escudero a Inglaterra, Tom Jones (1749) y Joseph Andrews (1742), cuyo subtítulo "Written in Imitation of the Manner of Cervantes, Author of Don Quixote" ya manifestaba "its dependence upon de Spanish model" (Borgmeier, 2005, p.52), muestran el interés de este escritor por Don Quijote de la Mancha $(1605,1615) .{ }^{5}$ La influencia de Cervantes en Henry Fielding fue objeto de valoración por parte de la crítica en los artículos de The Works of Henry

4 Pese a ello, factores como la rivalidad entre adversarios o el género del escritor influían en las valoraciones críticas emitidas. Los trabajos de mujeres no siempre se evaluaron de acuerdo a los mismos parámetros que se aplicaban para el análisis de las obras escritas por hombres. El declive de esta tendencia llegaría a finales de siglo cuando autoras como Mary Wollstonecraft (1759-1797) destacaron en la realización de tareas de revisión de textos y cuando se agudizó el principio de imparcialidad que aseguró que las mujeres y los hombres fueran tratados en condiciones de mayor igualdad (Donoghue, 1996: 160-161).

5 Queda fuera del alcance de este trabajo examinar la influencia de Cervantes en Roderick Random (1748) y Tom Jones (1749) dado que se publicaron antes de que vieran la luz The Monthly Review y The Critical Review. La reseña de la continuación de la novela de Henry Fielding, The History of Tom Jones the Foundling in his Married State (1749) (The Monthly Review 2, noviembre 1749: 25-26) carece de observación alguna acerca de que su autor, que la reseña no reconoce como Fielding, hubiera recurrido a la obra de Cervantes para su composición así como tampoco lo hace la valoración crítica de Amelia (1751) (The Monthly Review 5, diciembre 1751: 510-515). Tampoco los artículos de Peregrine Pickle (1751) (The Monthly Review 4, marzo 1751: 355-364) y Humphry Clinker (1771) (The Monthly Review 45, agosto 1771: 152; The Critical Review 32, agosto 1771: 81-88) hacen referencia alguna a la incorporación de elementos cervantinos en estos trabajos. 
Fielding, Esq; with the Life of the Author, publicados en 1762 (The Monthly Review 26, mayo 1762: 364-375; The Monthly Review 26, apéndice 1762: 481-494; The Monthly Review 27, Julio 1762: 49-56; The Critical Review 14, julio 1762: 1-21). Los evaluadores de The Monthly Review y The Critical Review seleccionaron, para incluir en sus respectivas reseñas, un mismo fragmento del prólogo de la obra que contenía la visión del biógrafo, Arthur Murphy, acerca de los tres periodos en la evolución del talento de Fielding a los cuales se les atribuía una correspondencia exacta con sus novelas Joseph Andrews, Tom Jones y Amelia. Este texto comunica al lector que Joseph Andrews había sido concebida para imitar el estilo y la manera de Cervantes y que Fielding había copiado "the humour, the gravity, and the fine ridicule of his master" de manera exquisita (The Monthly Review 26, apéndice 1762: 490; The Critical Review 14, julio 1763: 13). La cita incorpora, además, un elogio sobre el modo en que el personaje de Parson Adams había sido concebido. Su humanidad, benevolencia, bondad y celo por la virtud inspiraban el sentimiento de un fuerte apego hacia él. Su honestidad, talento, erudición, conocimientos de cultura clásica y de los escritos sagrados le hacían acreedor de toda estima y respeto, mientras que su sencillez e inocencia despertaban la sonrisa por el contraste que inyectaban con respecto a su verdadero carácter intelectual. Estas cualidades lo erigían en objeto de risa lejos de provocar la pérdida de respeto hacia él. Pero, sobre todo, era su distracción, su punto débil, lo que llevaba a este clérigo a convertirse en casi un rival del famoso Don Quijote. Las aventuras a las que le conducía este rasgo se impregnaban de un cierto aire romántico que también acompañaba al caballero andante (The Monthly Review 26, apéndice 1762: 491; The Critical Review 14, julio 1763: 13-14). El carácter que identificaba la personalidad de Adams muestra, tal y como queda representado en los artículos de prensa, una clara afinidad entre Don Quijote y Adams. ${ }^{6}$

6 Eric J. Ziolkowski también concuerda con esta visión. A su juicio, Fielding diseñó el personaje de Adams a imagen de don Quijote, con quien guardaba un parecido extraordinario en los rasgos más destacados de su carácter y a quien Fielding 
No obstante, estas no eran las únicas referencias a la tradición del quijotismo que se incorporan en el fragmento citado. En él se desvela, a su vez, que las situaciones a las que le llevaba su olvido tendían a provocar la risa al igual que hacían los errores del héroe español (The Monthly Review 26, apéndice 1762: 491; The Critical Review 14, julio 1763: 13-14).

El análisis de The Works of Henry Fielding prosigue con la inclusión de una nueva opinión de Arthur Murphy, en la que se vuelve a comparar la novela de Joseph Andrews con el Quijote. El biógrafo aseguraba que la aventura en la que el caballero andante confundía la bacía del barbero con el yelmo de Mambrino no era más ridícula ni verdaderamente más cómica que la que había vivido Parson Adams de viaje en Londres con el fin de vender un conjunto de sermones:

I will venture to say, that when Don Quixote mistakes the barber's bason for Mambrino's helmet, no reader ever found the situation more ridiculous and truly comic than Parson Adam's travelling to London to sell a set of sermons, and actually snapping his fingers and taking two or three turns round the room in extacy, when introduced to a bookseller in order to make an immediate bargain; and then immediately after, not being able to find those same sermons, when he exclaims, 'I profess, I believe I left them behind me' (The Monthly Review 26, apéndice 1762: 491; The Critical Review 14, julio 1763: 14).

La transcripción de estos fragmentos le servía a The Monthly Review y a The Critical Review para proporcionar información al lector acerca de Adams y para poner de manifiesto que en la configuración de este personaje se había seguido de cerca el modelo del Quijote, con quien se compara al tiempo que equipara.

percibía como una figura con una fuerte resonancia moral y religiosa que representaba las virtudes esenciales cristianas como la inocencia, caridad, benevolencia, sencillez y compasión. Ziolkowski ve en la religión cristiana una fuente indispensable de identidad de la figura de Adams (1991: 32-33). 
Asimismo, en las dos cabeceras se elogia el talento de Fielding y las cualidades de su obra, que caracterizan el segundo periodo en el que produce Tom Jones. En el texto proporcionado por la crítica se establece una relación comparativa entre su autor y Cervantes mediante la incorporación de un nuevo juicio de valor del biógrafo en el que se destaca la excelencia del novelista y dramaturgo inglés, a quien se reconoce como una eminencia en todos los puntos esenciales de la composición. Estas características, junto con su rica inventiva, imaginación sutil, juicio cultivado e ingenio vivaz, llevaban a declararlo el Cervantes inglés (The Monthly Review 27, julio 1762: 51; The Critical Review 14, julio 1763: 18). La cita de esta proclamación es una clara muestra de la admiración por la figura de Cervantes y un reconocimiento de que se encontraban ante uno de los grandes escritores de la historia inglesa:

[...]: and thus our author being confessedly eminent in all the great essentials of composition, in fable, character, sentiment, and elocution; and as these could not be all united in so high an assemblage, without a rich invention, a fine imagination, an enlightened judgment, and a lively wit, we may fairly here decide his character, and pronounce him the English Cervantes.

De la lectura de la reseña de Tristram Shandy (1759-67) se extraen una serie de características en las que se reconoce la huella del autor del Quijote en Laurence Sterne. El crítico de The Monthly Review inserta un extracto de la obra que alude a Cervantes y que refleja la opinión de Walter Shandy con respecto a la influencia de los nombres propios en las personas:

His opinion, in this matter, was, That there was a strange kind of magick bias, which good or bad names, as he called them, irresistibly impressed upon our characters and conduct.

The Hero of Cervantes argued not the point with more seriousness,-nor had he more faith,-or more to say on the powers 
of Necromancy in dishonouring his deeds,-or on Dulcinea's name, in shedding lustre upon them, than my father had on those of Trismegistus or Archimedes, on the one hand,-or of Nyky and Simkin on the other (21, apéndice 1759: 564).

En este fragmento se muestran al lector características comunes claramente apreciables entre el personaje de don Quijote y el del padre de Tristam. Al igual que Alonso Quijano, que había tratado de garantizar una nueva identidad a partir de la adopción del nombre de Don Quijote de la Mancha, Walter Shandy encontraba una estrecha relación entre la imposición de los nombres y las personas, a pesar de que había decidido llamar a su hijo Tristam, un nombre por el que sentía gran aversión (The Monthly Review 21, apéndice 1759: 566). Además de esta analogía con la figura del Quijote, el crítico, en su recorrido por la novela, selecciona un fragmento que da comienzo con una llamativa invocación y reconocimiento a Cervantes, aunque esta alusión no se percibe como sinónimo de influencia cervantina. Su extracción del mundo interno de la novela contribuye a ampliar el entendimiento de la herencia literaria así como la percepción de la relación y visión de Sterne con respecto a Cervantes, y es una clara evidencia de las referencias a este escritor en Tristam Shandy. Su aporte representa una nueva muestra del afecto que Sterne tenía hacia el consagrado escritor y permitía al público en general conocer algunos episodios de su vida, como la pérdida de movilidad de la mano izquierda. ${ }^{7}$

\section{INVOCATION}

Gentle spirit of sweetest humour, who erst didst fit upon the easy pen of my beloved CERVANTES; Thou who glided'st daily through his lattice, and turned'st the twilight of his prison into noon-day brightness by thy presence-tinged'st his little urn of water with heaven sent Nectar, and all the time he wrote of San-

7 En esta invocación el autor incluye una nota que explica que Cervantes había perdido el uso de la mano en la batalla de Lepanto. 
cho and his master, did'd cast thy mystic mantle o'er his wither'd* stump, and wide extended it to all the evils of his life- $[\ldots]$ (The Monthly Review 36, febrero 1767: 99).

En esta reseña se añade una nueva alusión a Cervantes cuando se trata sobre una cuestión relativa al humor pero, al igual que en el caso anterior, se deja atrás cualquier tipo de analogía con su obra. The Monthly Review no respalda los planteamientos de algunos coetáneos, basados en la comparación de la capacidad humorística de Sterne con la de Cervantes y Rabelais. La posición del autor difiere notablemente con la de aquellos que comparaban a Sterne con Cervantes en esta faceta y, aunque se aproximaba más a la línea de quienes comparaban su capacidad con la de Rabelais, sus apreciaciones le llevaban a afirmar que todos ellos estaban equivocados:

Several have compared Mr. Sterne, in his humorous capacity, to Cervantes; and others, with more propriety, to Rabelais; but they are all mistaken. The Reviewers have, at length, discovered his real prototype, Harlequin (The Monthly Review 36, febrero 1767: 93).

The Critical Review tampoco avala la visión de la época. El crítico observa una mayor proximidad entre Sterne y Rabelais, y apenas hace referencia alguna a la influencia del trazo alegre cervantino, aunque traslada al lector una declaración de Sterne en la que manifestaba su simpatía hacia el escritor francés así como su afecto hacia Cervantes, con la que se deja constancia de las abundantes alusiones al autor del Quijote en esta obra y de la admiración que Sterne sentía por él: "My dear Rabelais, and my dearer Cervantes!" (The Critical Review 11, abril 1761: 315). A su juicio, los rasgos más evidentes de la huella rabelaisiana en Tristam Shandy se aprecian en "the address, the manner, and colouring, [...] the same sort of apostrophes to the reader, breaking in upon the narrative, $[. .$.$] the same whimsical disgressions,$ and the same parade of learning" (The Critical Review 11, abril 
1761: 315). Con respecto a la afinidad entre Sterne y Cervantes, establece que "[ $t$ ]here is no more resemblance between his manner and that of Cervantes, than there is between the solemnity of a Foppington and the grimace of a Jack Pudding" (The Critical Review 11, abril 1761: 315). Aún a pesar de los diversos puntos de comparación que encuentra entre Sterne y Rabelais, asegura que se veía en la obligación de reconocer que existían algunos golpes de humor en Tristam Shandy que trascendían al autor francés (The Critical Review 13, enero 1762: 67), una opinión que parece traslucir que el humor de Sterne era un rasgo único que lo distinguía de otros escritores. ${ }^{8}$

Los numerosos incidentes humorísticos, las acertadas observaciones sobre la vida y los personajes, así como el gusto y la erudición que impregnan esta novela son reconocidos por The Critical Review, a pesar de que se concibe como digresiva e incoherente (The Critical Review 11, abril 1761: 316). De hecho, su significado era tan indeterminado e incierto que apenas se podía analizar el contenido (The Critical Review 21, febrero 1766: 141). Tristam Shandy supuso un desafío a la crítica, que se encontró ante la duda de si censurar más la obra como impertinente o si, por el contrario, elogiar sus características más sobresalientes y su calidad: "We know not whether most to censure the impertinence, or commend the excellencies of this strange, incongruous, whimsical performance" (The Critical Review 13, enero 1762: 69). The Monthly Review expresa una valoración positiva de los dos primeros volúmenes, que recomienda al público, calificándolos de entretenidos y como una fuente de humor genuino (The Monthly Review 21, apéndice 1759: 571), pero la visión cambia completamente a partir de los siguientes libros. La nueva reflexión crítica establece que se encontraban ante una obra absolutamente aburrida y escandalosamente indecente, con un humor lascivo y obsceno, y ante unos personajes que ya no eran ni sorprendentes

8 Autores como Thomas Seccombe (1909: 186) o Felicitas Kleber (2005: 80) no reconocen el humor de Sterne como exclusivamente rabelaisiano sino Sternean. 
ni singulares (The Monthly Review 24, febrero 1761: 103-104, 109, 116). En general, esta cabecera literaria cuestiona la violación del decoro, totalmente inapropiado para un clérigo como Sterne (The Monthly Review 26, enero 1762: 31-32), y no aprecia la singularidad de la obra, que se describe como abigarrada y se denomina "the pantomime of literature" (The Monthly Review 36, febrero 1767: 93). The Monthly Review es un ejemplo de que el decoro fue valorado por la crítica a la hora de emitir su juicio. Tal y como establece Alan B. Howes, la moralidad influyó en la recepción de Sterne. Así, para algunos críticos el conocimiento de su profesión determinó la manera en que la obra fue valorada mientras que otros excusaron los actos que transgredían los límites del decoro profesional (Howes, 1995: 6).

El examen de la influencia de Don Quijote en la literatura británica lleva al análisis de la reseña de la novela The Adventures of Sir Launcelot Greaves (1762), de Tobias Smollett, debido al grado de similitud que se advierte entre ambas en la valoración proporcionada por The Critical Review. En ella se muestra al lector que el talento de Cervantes, a quien se describe como un autor digno de admiración, fue reconocido por Smollett, que se inspiró en Don Quijote para la creación de varios de sus personajes principales. El influjo que había ejercido en su obra se deja sentir en Sir Launcelot y en Crabshaw, que parecían estar basados en las figuras del caballero de la Mancha y de su ingenioso escudero Sancho Panza, respectivamente. No obstante, apunta el crítico, los personajes de Sir Launcelot Greaves no eran copias idénticas de ellos. Se parecían, sin duda, pero no eran imitaciones. Más allá de esta influencia cervantina, hace un reconocimiento al talento de Smollett cuando afirma que los lectores que no estaban familiarizados con Don Quijote y Sancho estarían encantados con Sir Launcelot y Crabshaw, mientras que aquellos que sí lo estaban estarían sorprendidos ante la posibilidad de aportar originalidad a personajes conformados siguiendo aquel modelo: 
Readers unacquainted with the don and his Squire, will be delighted with Sir Launcelot and Crabshaw; those who have attended the mirror of chivalry through the course of his strange adventures, and listened with wonder to the shrewd remarks of Sancho, will be surprised at the possibility of giving originality to characters formed on that model (The Critical Review 13, mayo 1762: 428).

Al contrario que The Critical Review, The Monthly Review no establece ningún tipo de analogía entre The Adventures of Sir Launcelot Greaves y el Quijote. En la valoración de apenas dos líneas proporcionada por el crítico se comenta desfavorablemente que es un trabajo mejor que cualquier novela común pero impropio de la pluma de Smollett: "Better than the common Novels, but unworthy the pen of Dr. Smollet" (The Monthly Review 26, mayo 1762: 361). En palabras de Frank Donoghue, la posición de Smollett como rival de Ralph Griffiths ejerció una influencia poderosa sobre el trato que recibió este escritor en de The Monthly Review. A su juicio, la tendencia de esta publicación fue la de mermar la reputación de Smollett quizás con la intención de que se tomara menos en serio la cabecera en la que era editor (1996: 127).

La asimilación de la novela de Cervantes se pone de manifiesto en el mundo de la ficción en otros textos producidos en la segunda mitad de siglo tales como The Female Quixote; or, the Adventures of Arabella (1752), Tarrataria; or, Don Quixote the Second (1761), The Spiritual Quixote; or, the Summer's Ramble of Mr. Geoffrey Wildgoose (1773), The Philosophical Quixote; or, Memoirs of David Wilkins (1782), The City Quixote (1785), The Amicable Quixote; or, the Enthusiasm of Friendship (1788), William Thornborough, the Benevolent Quixote (1791) y The History of Sir George Warrington; or, the Political Quixote (1797). ${ }^{9}$ Estas narrativas son ficciones

9 Las revisiones de Tarrataria; or, Don Quixote the Second (1761) (The Monthly Review 24, mayo 1761: 353; The Critical Review 11, mayo 1761: 412) y de The City Quixote (1785) (The Critical Review 59, marzo 1785: 235) no contienen alusión alguna a su relación con el Quijote aunque en ellas se indica que sus autores se habían valido del humor y, en el caso de Tarrataria, también de la sátira y del ingenio para su configuración. 
quijotescas en tanto que relatan "las aventuras de un personaje de psicología similar inspirada en la de don Quijote: [...]" (Garrido, 2014: 25) y constituyen un claro ejemplo de cómo el término quixote entró a formar parte del léxico inglés. ${ }^{10}$ En ellas se hace uso de la parodia con el fin de emitir juicios y una visión satírica de la sociedad británica así como de "establecer un diálogo con géneros narrativos anteriores $\mathrm{y}$, al mismo tiempo, cuestionar sus limitaciones o fallos" (Borham, 2015: 12).

La huella de Don Quijote se hace visible en una obra protagonizada por una mujer quijote: The Female Quixote; or, the Adventures of Arabella, de Charlotte Lennox. En abril de 1752, The Monthly Review ponía de manifiesto que esta novela constituía un ejemplo representativo de la influencia cervantina en la literatura británica. En su exposición, el crítico explica que, así como las aventuras del caballero andante se habían escrito para exponer los disparates de los romances caballerescos, ${ }^{11}$ las aventuras de la heroína inglesa se habían diseñado para ridiculizar el amor romántico y mostrar la tendencia que tenían los libros de caballerías de influir en el comportamiento de las personas, incluso en el de sus lectoras (The Monthly Review 6, abril 1752: 249). Se presenta aquí una analogía entre Don Quijote y The Female Quixote en tanto que la lectura en exceso de romances llevaba a los lectores, entre los que se encontraban las mujeres, a sufrir alucinaciones fácilmente, a pesar de que Arabella no había llegado tan lejos como para con-

10 Otros textos que hacen constar en su título la palabra "quixote" se publicaron ya entrado el siglo XIX. Véase el caso de The Infernal Quixote. A Tale of the Day (1801), de Charles Lucas, y The Political Quixote; or, the Adventures of the Renowned Don Blackibo Dwarfino, and his Trusty Squire, Seditiono (1820).

11 Los conceptos novel y romance eran empleados indistintamente por los críticos para referirse a obras de ficción narrativa dado que su distinción aún no estaba bien establecida por aquel entonces. En The Progress of Romance (1785) Clara Reeve establece que el romance se centra en lo que no ha ocurrido ni es probable que ocurra nunca mientras que la novela ofrece una relación familiar de esas cosas tal y como suceden cada día ante nuestros ojos (1930: 111). Ioan Williams indica que esta distinción fue el resultado de un largo proceso de desarrollo (1970: 4). 
fundir un rebaño de ovejas con un ejército de hombres o como para identificar molinos de viento con gigantes (The Monthly Review 6, abril 1752: 249). Así pues, por un lado, se pone de relieve la sátira de las virtudes y modelos caballerescos y, por otro, se presentan al lector claras características quijotescas en Arabella cuyo comportamiento venía determinado por los libros que había leído, al igual que le había sucedido a don Quijote. En esta valoración se encuentra implícito que Lennox hizo uso del modelo creado por Cervantes, cuyo famoso hidalgo había enloquecido por la lectura excesiva de libros de caballerías, al tiempo que se advierte del papel de las mujeres como consumidoras de productos culturales en aquella época.

El quijotismo de Arabella encuentra su origen en los conocimientos adquiridos en un lugar bastante apartado del país a través de la lectura de narrativas romancescas que la habían llevado a interiorizar la creencia distorsionada de que las situaciones sobre las que había leído eran imágenes fidedignas de la realidad y que los personajes que estaban representados en los libros no eran otros sino aquellos con los que se encontraría en la vida real (The Monthly Review 6, abril 1752: 250). El crítico traslada a los lectores las nociones románticas y el comportamiento extravagante de esta heroína en la cita de un pasaje cuyo contenido cree que es más que suficiente para hacerse una idea de cuál era su concepto romántico sobre la caballerosidad (The Monthly Review 6, abril 1752: 255). La información seleccionada detalla que el padre de Arabella había decidido dar a su hija en matrimonio a Granville. Pero, así como él pronto se apasionaría por ella, ella sentiría una fuerte aversión hacia él. Sus marcadas convenciones románticas la llevarían a rechazar su declaración de amor que no se había correspondido en absoluto con las pautas de conducta que cabía esperar y que había constituido una gran ofensa para ella (The Monthly Review 6, abril 1752: 250-255). El crítico reconoce que Arabella esperaba ser tratada con profundo respeto por parte de sus admiradores y añade que, así como don Quijote siempre encontraba hazañas dignas de su va- 
lentía caballeresca, ella tampoco perdía la oportunidad de mostrar su verdadero espíritu heroico como castigo a amantes presuntuosos. Pone de ejemplo a Mr. Harvey, un caballero a quien veía ocasionalmente en la iglesia, y al pobre Edward, que había adoptado el disfraz de noble aunque en verdad no era más que un trabajador empleado por el jardinero de su padre, quienes habían sido víctimas de los peculiares rasgos románticos que la caracterizaban (The Monthly Review 6, abril 1752: 255). Como el caballero andante, la heroína inglesa traslada a la realidad las historias leídas en los libros, propiciando un sin fin de situaciones realmente extravagantes y cómicas. Todas estas similitudes percibidas por la crítica constituyen ejemplos de un modelo de quijotismo femenino en la literatura británica del siglo XVIII. La importancia de este Quijote femenino como fuente de un nuevo arquetipo quijotesco también ha sido reconocida por autores como Pardo García (2004), Garrigós González (2009), Amy Pawl (2009) y Borham Puyal (2015).

El humor y las extravagancias presentes en esta novela se muestran en la lectura de un nuevo fragmento que el crítico extrae del texto con la intención de dar a conocer al lector el convencimiento de Arabella de que Mr. Selvin estaba enamorado de ella porque suponía que todos los hombres que la veían eran admiradores suyos. Esta creencia la había llevado a desterrarlo de su presencia y había desencadenado una serie de situaciones cómicas que ponían de manifiesto su locura quijotesca causada por su interpretación de los romances. Este episodio refleja, además, destacadas analogías entre los personajes de Sancho y Lucy porque, semejante al caso del escudero, ella también tenía dificultad para recordar palabras. Como en él se relata, Lucy había incurrido en un error al transmitir un mensaje de Arabella. Su equivocación la había hecho emplear el término solation para referirse a consolation (The Monthly Review 6, abril 1752: 260). El mal uso de esta palabra permite tomar conciencia de sus defectos de dicción y equipararlos a las alteraciones que Sancho también provocaba en la forma de algunos vocablos. 
La influencia de Don Quijote en la obra de Lennox se aprecia en otra equivalencia que el crítico establece entre el hidalgo y la heroína quijotesca así como entre la criada y el escudero. En este caso, y ya en la parte final de la reseña, resalta que el caballero andante hablaba siempre juiciosamente en cada ocasión donde la caballerosidad estaba fuera de cuestión y que lo mismo ocurría con Arabella, excepto cuando se trataba del amor y de la cortesía (The Monthly Review 6, abril 1752: 261-262). Apunta, a su vez, que la sencillez de modales estaba presente tanto en Sancho como en Lucy, por no mencionar el apoyo tan evidente que ambos brindaban a las flaquezas del caballero y de la heroína (Monthly Review 6, abril 1752: 262). A su juicio, todos los parecidos entre los personajes de don Quijote y Arabella y de Sancho y Lucy habían sido provocados por Lennox lo cual es una clara muestra de la huella cervantina en esta autora. El crítico concluye el artículo con la locución latina nota bene [nótese bien] en la que se resalta que el autor de la publicación Covent Garden Journal no había tenido ningún recelo en preferir este trabajo, en muchos sentidos, al del inimitable Don Quijote (The Monthly Review 6, abril 1752: 262).

La evaluación de The Spiritual Quixote proporcionada en The Critical Review otorga en sus primeras líneas un claro reconocimiento a la obra de Cervantes. En ella se hace mención al hecho de que es raro que trabajos que habían alcanzado una prestigiosa reputación no hubieran sido objeto de multitud de imitaciones, como era el caso del Quijote, que había sido admirado en toda Europa y motivado a varios autores a seguir los pasos de su ilustre autor. A modo de ejemplo, se citan tres trabajos: The Female Quixote, de Charlotte Lennox, Historia del Fray Gerundio, publicado en España en 1758 por el Padre Isla, y The Spiritual Quixote; or the Entertaining History of Don Ignatius Loyola, founder of the Order of Jesuits (1754), traducción al inglés de Histoire de l'admirable Don Iñigo de Guipúzcoa (1736) (The Critical Review 35, abril 1773: 275). El crítico de The Monthly Review también hace referencia a esta última producción y señala que el autor de la obra que estaba 
siendo reseñada probablemente la habría titulado de otro modo de haber sabido que unos veinte años atrás ya se había publicado un trabajo en aquel país con un título similar (The Monthly Review 48, mayo 1773: 388).

La prensa literaria pone de manifiesto que la parodia de Cervantes motivó a Richard Graves para crear una novela satírica dirigida contra los metodistas. El producto fue The Spiritual Quixote que, según The Critical Review, tiene como objetivo satirizar los proyectos visionarios de esta corriente religiosa con cuyas doctrinas se deleitaba el protagonista, Geoffrey Wildgoose. La información contenida en el artículo establece que este movimiento se había originado en Inglaterra alrededor de 1730, cuando John Wesley, su hermano Charles, Mr. Clayton y otros jóvenes de la universidad de Oxford, a quienes se uniría dos años más tarde Whitefield, formaron una sociedad que, en muy poco tiempo, se convertiría en una epidemia social, influyendo intensamente en la vida de aquellos individuos que padecían alguna enfermedad mental o debilidad de entendimiento:

The design of the work now before us is to ridicule and expose the fanatical notions, the rambling humour, and the visionary projects of our modern itinerant reformers, the Methodists; a species of folly which, ever since the year 1736, has infected almost every person labouring under a disorder of the brain, or impotency of understanding (The Critical Review 35, abril 1773: 276).

Sostiene el crítico que los principios puritanos que el protagonista había internalizado lo habían preparado para deleitarse con las doctrinas de los metodistas (The Critical Review 35, abril 1773: 278). La lectura de diferentes discursos y tratados religiosos, que diferían bastante en sus principios pero que coincidían en su naturaleza en contra de la iglesia de Inglaterra, había tenido un efecto tan notable en Wildgoose que le había desestabilizado su mente y hecho perder casi el juicio. A esas lecturas habría que sumarle, prosigue el crítico en su valoración, la la- 
bor de los predicadores itinerantes y de las reuniones nocturnas que tenían lugar no muy lejos de su casa y a las que también asistía. Su inclinación piadosa de trasladar las doctrinas que había escuchado en esas asambleas, los prejuicios que había concebido contra el vicario de la parroquia y la propensión natural del individuo de propagar sus opiniones le habían instado enérgicamente a dar rienda suelta a todos los conocimientos de ámbito espiritual que había adquirido (The Critical Review 35, abril 1773: 278-279).

La reseña incorpora una serie de pasajes del libro para darle al lector una idea anticipada de su contenido. En uno de ellos se da a conocer la decisión de Wildgoose de abandonar su casa $\mathrm{y}$, como un verdadero quijote espiritual, poner todo su empeño en reavivar las doctrinas de la Reforma haciéndose misionero y predicando sus nociones religiosas por todas las partes del reino, en imitación de Mr. Whitefield y sus asociados. Emprende su viaje en busca de aventuras espirituales en compañía de su particular escudero, su amigo zapatero Jerry Tugwell (The Critical Review 35, abril 1773: 280). En el curso de sus peregrinaciones visitan, entre otras, las localidades de Gloucester, Bath, Bristol, Cardiff, Newport, Worcester y Warwick, en las que se encuentran con abundantes y ridículos desastres e incidentes fabulosos que, en opinión del crítico, estaban relatados de manera sencilla y con humor (The Critical Review 35, abril 1773: 280-281). Otro de los pasajes seleccionados por el crítico describe un accidente ocurrido en unas carreras en Warwick, donde un joven a caballo lanzó, con gran vehemencia, un decantador a la cabeza del protagonista. Este incidente, que se considera como debidamente calculado para sosegar la mente, junto con las conversaciones del respetable clérigo Grenville y de Miss Townshend, de quien se enamora el héroe, le incitan a abandonar la empresa que había comenzado, a regresar a casa, donde se establece, y a casarse con su heroína (The Critical Review 35, abril 1773: 285-286).

The Monthly Review comienza su valoración con un reconocimiento de la singularidad de The Spiritual Quixote, una obra 
diferenciada de las novelas basura que abundaban en el mercado en aquel momento, a pesar de que la pobreza del tema planteado con las aventuras de un entusiasta fanático, tal y como se describe al predicador metodista, era impropia del talento del autor (The Monthly Review 48, mayo 1773: 384). ${ }^{12}$ El análisis proporcionado por The Monthly Review es prueba igualmente de que el Quijote fue un modelo a seguir en esta novela y de cómo Richard Graves aplicó el propósito quijotesco de Cervantes a su personaje Wildgoose, quien opta por comenzar una nueva vida de caballero andante y contribuir, de ese modo, a la propagación de la nueva corriente religiosa por la que se sentía enormemente atraído. La relación del protagonista con el personaje cervantino y su escudero se hace evidente cuando el crítico establece que Wildgoose se había convertido en un predicador metodista que había atraído a un zapatero para que fuera su Sancho espiritual (The Monthly Review 48, mayo 1773: 384). Tras una muestra de algunos de los pasajes más destacados de la novela, concluye el análisis con una relación de los motivos por los que el protagonista había recobrado el juicio y que, al igual que The Critical Review, se resumen en el golpe que había recibido en la cabeza mientras estaba predicando en una carrera de caballos, en la sensata conversación de un clérigo y en el amor sincero hacia una mujer respetable que no era amiga de su quijotismo. En esta conclusión se hace una apreciación positiva del personaje cuando finalmente se recupera de su locura y es feliz. El crítico no duda en afirmar que la manifestación de su cordura le causaría una enorme satisfacción al lector benevolente (The Monthly Review 48, mayo 1773: 387). El conflicto entre la pasión y la razón se hace evidente en una época de progreso hacia un pensamien-

12 El éxito obtenido por Richardson, seguido inmediatamente por el de Fielding y Smollett, cambió de manera radical la actitud de los críticos con respecto a la novela. La respuesta del público a las grandes novelas de la década de los cuarenta y cincuenta fomentó la producción de numerosas obras de poca calidad, que fueron cuestionadas por la crítica que además mostró su preocupación por los efectos indirectos de trabajos que podían ser considerados inofensivos o moralmente beneficiosos (Williams, 1970: 9-11). 
to ilustrado que se fundamentaba principalmente en el racionalismo. En Carta sobre el entusiasmo, el escritor y filósofo inglés Anthony Ashley Cooper, conde de Shaftesbury (1671-1713), argumenta que la religión es una causa de pánico en situaciones de entusiasmo mayor de lo normal pero que puede ser mitigado por la razón y por el buen humor (1997: 104-106). A su juicio, el buen humor no es únicamente el antídoto contra el entusiasmo sino también "el mejor fundamento de la piedad y la verdadera religión" (1997: 111). De ahí que Shaftesbury, tal y como establece Cerezo Galán, “tuviera en tan alta estima a Cervantes por su fina ironía, capaz de poner en ridículo toda política del entusiasmo." (2006: 230).

La continuación dieciochesca del Quijote se explicita en el título de otra quixotic fiction, The Philosophical Quixote, en la que se vuelve a introducir una figura quijotesca como protagonista. De acuerdo con la crítica, esta novela está diseñada para hacer una sátira sobre ciertas aventuras extravagantes y descubrimientos filosóficos de la época y sobre los intentos de aplicarlos en el ámbito de la medicina (The Critical Review 54, diciembre 1782: 438; The Monhtly Review 68, marzo 1783: 273). En esta ocasión, el héroe, David Wilkins, se describe como un boticario ávido de realizar algún descubrimiento científico. En su afán, realiza experimentos constantemente, trasladando a cada uno de ellos las fantasías de su propia imaginación. Con este quijotismo filosófico, el autor de The Critical Review señala que Wilkins se muestra como una persona generosa, bondadosa y humana, siempre a disposición de los deseos y sufrimientos del prójimo, a no ser que se presentara algún descubrimiento. De ahí que sea siempre respetable y digno aunque, a menudo, ridículamente absurdo (The Critical Review 54, diciembre 1782: 438). La reseña hace un guiño al Quijote en la cita de un fragmento de la obra donde se narra un experimento llevado a cabo por Wilkes relacionado con la electricidad y en el que se encuentra una equivalencia con el caballero de la Mancha: 
We wish to give a specimen of this pleasing performance, and, as the common doctrines of electricity are generally known, shall select one of the windmills of his new Quixote (The Critical Review 54, diciembre 1782: 439).

En la valoración de The Philosophical Quixote proporcionada por The Monthly Review no se hace referencia alguna a la relación de esta obra con el Quijote ni tampoco se evalúa de manera tan favorable como en la reseña anterior. El crítico resalta que la novela contribuye poco a despertar el interés de los lectores en general y menos aún a procurar su entretenimiento. Sobre el autor se establece que parecía ser un hombre de ciencia aunque las reflexiones que trasladaba al público eran poco originales y vulgares al igual que los incidentes, que habían sido imaginados y descritos de manera deficiente:

[t]he ridicule of it is only calculated to strike profesional men. There is little in it to interest general readers, and still less to amuse them. The author appears to be a man of science. In other respects his invention is languid; his reflections are trite and superficial; and the incidents interspersed in his work are low vulgar, weakly imagined, and poorly described (The Monhtly Review 68, marzo 1783: 273).

La figura del Quijote se emplea también en The Amicable Quixote. La crítica pone de relieve la falta de naturalidad de muchas situaciones narradas en esta producción, a pesar de haber tenido en cuenta el quijotismo para la realización de esta valoración. Algunos personajes eran sumamente cuestionables dado que parecían de caricatura más que sacados de la vida real (The Monthly Review 80, enero 1789: 61). The Critical Review facilita un juicio prácticamente coincidente. El crítico comparte las apreciaciones anteriores y resalta que la obra presenta muchos errores tanto en la trama como en los personajes y en los acontecimientos. A su juicio, y a pesar de que en ocasiones los personajes podían resultar interesantes, su quijote era cuestionable: The Quixote, 
eager in pursuit of friendship is not detailed at length, or sufficiently brought forward." (The Critical Review 67, enero 1789: 77).

En la década siguiente, nuevos quijotes han quedado representados en las novelas satíricas William Thornborough, The Benevolent Quixote y The History of Sir George Warrington; or, the Political Quixote. En el análisis de The Benevolent Quixote proporcionado por The Critical Review se ofrece una valoración positiva de esta obra, que entrega una buena dosis de entretenimiento y disfrute al lector. El autor es elogiado por su representación de William Thornborough, a quien se describe como un personaje amigable, respetable y atractivo, con quien se conecta a nivel emocional (The Critical Review ser2 v1, abril1791: 471). The Monthly Review también hace referencia al quijotismo presente en este trabajo pero cuestiona que el novelista buscara provocar la risa a expensas de hombres buenos, honestos y benévolos, quienes raras veces fallaban a la hora de transmitir la idea de debilidad de mente y de corazón, y quienes se encontraban, normalmente, en el lado más terrible de las transacciones mundanas. Asimismo, tampoco aprueba que la obra hubiera sido concebida para comprobar tales cualidades en gente pudiente como el protagonista, William Thornborough, un hombre en posesión de una gran fortuna, heredada tras la muerte de su tío, su padre y un hermano mayor, que había tomado la firme determinación de emplear su tiempo y su dinero en hacer todo el bien posible a aquellas personas que estuvieran en apuros (The Monthly Review ser 2 v4, febrero 1791: 229-230). Este propósito nada plausible le restaba méritos a la novela así como a la figura quijotesca.

La parodia en esta obra se presenta también con claras diferencias con respecto al Quijote. Mientras que el texto cervantino se constituye, ante los ojos del crítico, como una crítica burlesca real dado que su protagonista había dejado su casa como juez supremo del bien y del mal, y se había comprometido a eliminar la opresión en el mundo cuando esas nociones estaban aún vigentes, la sátira de The Benevolent Quixote no tenía ningún fundamento. Esta argumentación se respalda con la prueba de que 
en aquella época apenas se escuchaba hablar de la sensibilidad adoptando una dirección tan caritativa. De esta manera, el quijotismo se asocia con una visión anacrónica y carente de sentido, que no se correspondía ni tampoco estaba bien integrada en el pensamiento general de aquel momento.

Quixotism should have a manifest absurdity for its basis; and the original Don Quixote, a man who sallied forth as the supreme arbiter of right and wrong, who undertook to crush oppression, and to redress all injuries by force, was, during the prevalence of such wild notions, a true burlesque: but is it at this time a fashionable error, among young men of affluence, to dissipate their substance at the call of every real or pretended object of distress? We seldom hear of sensibility, however fashionable the word is grown, taking such a charitable direction; and we rather fancy that sensibilities of a more confined nature, put in prior claims, that sufficiently guard the heads of our young gentry against the dangers of indiscriminate liberality. So that this is a satire without an object, and of course a strained effort at novelty (The Monthly Review ser 2 v4, febrero 1791: 230).

Estas son las objeciones expresadas por el crítico sobre una obra que consideraba una de las más divertidas que había leído recientemente y cuyos personajes y descripciones, en ocasiones, le recordaban el trabajo titulado History of Pompey the Little (1751). La reseña concluye con la sospecha de que tanto The Benevolent Quixote como The Amicable Quixote habían sido escritas por el mismo autor debido a la similitud existente entre los títulos y el propósito de ambas producciones (The Monthly Review ser.2 v.4, febrero 1791: 230). ${ }^{13}$

The Critical Review comienza su análisis de The History of Sir George Warrington; or, The Political Quixote con una cita de la novela en la que se describe al héroe como un hombre joven de gran fortuna (The Critical Review ser.2, v.23, mayo 1798: 112-113). En

13 Bautista Naranjo establece que las hermanas Elizabeth y Jane Purbeck son las autoras confirmadas de William Thornborough, The Benevolent Quixote (2015: 193). 
la imagen que se ofrece de este personaje se establece una analogía con don Quijote dado que se había aficionado a la lectura como consecuencia de un aislamiento que le había sido impuesto tras una caída de un caballo. El crítico detalla que su quijotismo político había sido motivado por un trabajo de Thomas Payne (1737-1809), titulado Rights of Man (1791), que había encontrado entre los libros que el vicario de la parroquia había puesto en sus manos. A partir de aquel momento, se había declarado a sí mismo un paladín de la libertad y de la igualdad universal. Tras esta parte descriptiva de la reseña, se inserta una apreciación valorativa en la que se reconoce que la obra no contiene ningún elemento que deprave el conocimiento o corrompa el corazón y se indica que en ella se realizan algunas observaciones razonadas sobre el carácter, las actitudes y los principios propios de la época (The Critical Review ser.2 v.23, mayo 1798: 113-114).

Los datos recogidos en los estudios de las novedades bibliográficas proporcionadas por The Monthly Review y The Critical Review han confirmado el reconocimiento que la crítica otorgó a la influencia de Cervantes así como la excelente acogida de su obra en la literatura británica durante la segunda mitad del siglo XVIII. Estas dos cabeceras constatan que el Quijote es el texto cervantino que adquirió mayor repercusión en la prosa literaria de aquel periodo. Su impronta se refleja en las reseñas de destacadas obras de autores como Lennox, Fielding, Sterne y Smollett, cuyas novelas han contribuido a perpetuar la fama internacional de Cervantes y la inmortalidad de sus personajes, así como en las valoraciones de las ficciones quijotescas en las que se empleó la figura del Quijote como retrato satírico de la sociedad. En este sentido, se puede argumentar que los artículos analizados dejan constancia de la identificación por parte de la crítica de la presencia del texto cervantino en las producciones de autores británicos ya en aquella centuria. Además, la difusión de evaluaciones que mostraban al lector la conexión existente entre la novela británica y Cervantes supuso un importante estímulo para el autor en el reconocimiento de su talento y alcance literario así como una 
forma de acercamiento de su obra al público. Esto incidió probablemente en la configuración del canon personal del lector e iría acompañado de la instalación de Cervantes no sólo en el canon crítico sino también literario de la época, cuando la destacada posición que The Monthly Review y The Critical Review habían adquirido en la historia de la crítica literaria en Gran Bretaña llevó a que se convirtieran en un referente en la formación del canon.

\section{Referencias bibliográficas}

\section{Fuentes hemerográficas}

A Literary Journal (1730-1731)

Analytical Review (1788-1799)

Anti-Jacobin Review (1798-1810)

British Critic (1793-1826)

Champion (1739-1742)

Covent Garden (1752)

Criterion (1922-1939)

Critical Review (1756-1817)

Daily Gazetteer (1735-1745)

General Magazine and Impartial Review (1787-1792)

General Review (1752)

Edinburgh Magazine and Review (1773-1776)

Edinburgh Review (1803-1929)

English Review (1783-1796)

Examiner (1710-1714)

Jacobite's Journal (1747-1748)

History of the Works of the Learned (1737-1742) 
Literary Journal (1744-1749)

Literary Magazine (1735-1736)

Literary Magazine; or Universal Review (1756-1758)

London Mercury (1919-1939)

London Review of English and Foreign Literature (1775-1780)

Memoirs of Literature $(1710-1714 ; 1717)$

Monthly Review (1749-1845)

National Review (1883-1950)

New Memoirs of Literature (1725-1727)

New Review (1782-1786)

Present State of the Republick of Letters (1728-1736)

Rambler (1750-1752)

Review (1704-1713)

Saturday Review of Politics, Literature, Science and Art (1855-1931)

Spectator (1711-1712)

Tatler (1709-1711)

Theatrical Review (1763)

True Patriot (1745-1746)

Weekly Magazine and Literary Review (1758)

\section{Referencias bibliográficas}

Acosta Aide, S. (1985): “El influjo de El Quijote en Joseph Andrews", Revista Canaria de Estudios Ingleses 11, pp. 69-80.

Ballesteros, I. (1989): “La presencia de Don Quijote de Cervantes en Joseph Andrews de Fielding, Anales Cervantinos 27, pp. 215-224. 
Barrio Marco, J. M. y Crespo Allué, M. J. (2007): La huella de Cervantes y del Quijote en la cultura anglosajona, Valladolid, Universidad de Valladolid.

Basker, J. (2005). "Criticism and the Rise of Periodical Literature" en Nisbet, H. B. y Rawson C., The Cambridge History of Literary Criticism, Vol. 4, The Eighteenth Century, Cambridge, Cambridge University Press, pp. 316-332.

Bautista Naranjo, E. (2015): La recepción y reescritura del mito de Don Quijote en Inglaterra (siglos XVII-XIX), Madrid, Dykinson.

Borgmeier, R. (2005): “Henry Fielding and his Spanish Model: 'Our English Cervantes'”' en Fernández-Morera, D. y Hanke, M., Cervantes in the English Speaking World, Kassel, Reichenberger, pp.43-64.

Borham Puyal, M. (2015): Quijotes con enaguas. Encrucijada de géneros en el siglo XVIII británico, [s.l.], JPM Ediciones.

Cerezo Galán, P. (2006): "Las figuras quijotescas de la melancolía", en Duque, F. et al, El yo fracturado. Don Quijote y las figuras del Barroco, Madrid, Círculo de Bellas Artes, pp. 211-262.

Cranfield, G. A. (1978): The Press and Society. From Caxton to Northcliffe, New York, Longman.

Donoghue, F. (1996): The Fame Machine: Book Reviewing and Eighteenth-Century Literary Careers, Stanford, CA, Stanford University Press.

Garrido Ardila, J. A. (2016): “Las rutas del Quijote por la novela inglesa del siglo XVIII", Cuadernos de estudios del siglo XVIII 26, pp. 17-31.

---. (2014): Cervantes en Inglaterra: el Quijote y la novela inglesa del siglo XVIII, Alcalá, Universidad de Alcalá.

---. (ed.), (2009): The Cervantean Heritage. Reception and Influence of Cervantes in Britain, Oxford, Legenda, Modern Humanities Research Association. 
---. (2006): “Un ejemplo señero de la Cervantean fiction: Roderick Random de Tobias Smollett", Bulletin of Hispanic Studies 83.5, pp. 133-155.

---. (2002): "La teoría cervantina de la novela en Roderick Random", Bulletin of Spanish Studies 79.5, pp: 543-562.

---. (2001a): “Cervantes y la Quixotic Fiction: sucesión episódica y otros recursos narrativos", Cervantes: Bulletin of the Cervantes Society of America 21.1, pp: 43-65.

---. (2001b): “Cervantes y la Quixotic Fiction: el hibridismo genérico", Cervantes: Bulletin of the Cervantes Society of America 21.2, pp: 5-26.

---. (1998): “Cervantes y la Quixotic Fiction: la parodia de géneros", Anales cervantinos 34, pp: 145-168.

Garrigós GonzÁlez, C: (2009). “¿Lecturas peligrosas o mujeres alienadas? Quijotismo femenino, locura y metaliteratura", en Hagedorn H. C., Don Quijote, cosmopolita: nuevos estudios sobre la recepción internacional de la novela cervantina, Cuenca, Ediciones de la Universidad de Castilla-La Mancha, pp. 307-322.

Godden, G. M. (2008): Henry Fielding: A Memoir, Boston, IndyPublish.

Graham, W. (1930): English Literary Periodicals, New York, Thomas Nelson and Sons.

Griffin, D. (2014): Authorship in the Long Eighteenth Century, Newark, University of Delaware Press.

HaIG, R. L. (1960): The Gazetteer 1735-1797: A Study in the Eighteenth-Century English Newspaper, Carbondale, Southern Illinois University Press.

Howes, Alan B. (1995): Laurence Sterne. The Critical Heritage, London y New York, Routledge.

Keeran, P. y Bowers, J. (2013): Literary Research and the British Eighteenth Century. Strategies and Sources, Plymouth: The Scarecrow Press. 
Kleber, F. (2005): “Laurence Sterne's Tristam Shandy and Don Quixote", en Fernández-Morera, D. y Hanke, M., Cervantes in the English Speaking World, Kassel, Reichenberger, pp. 65-80.

Marr, G. S. (1971): The Periodical Essayists of the Eighteenth Century with Illustrative Extracts from the Rarer Periodicals, New York, Sentry Press.

Müllenbrock, H. J. (1999): “Don Quijote and Eighteenth-Century English Literature", en Antor, H. y Cope, K. L., Intercultural Encounters: Studies in English Literature, Heidelberg, Universitatsverlag C. Winter, pp.197-209.

Pardo García, P. J. (2007): “El Siglo de Oro del Quijote en la literatura inglesa, 1740-1840", en Barrio Crespo, J. M. y Crespo Allué, M. J., La huella de Cervantes y del Quijote en la cultura anglosajona, Valladolid, Universidad de Valladolid, pp. 133-158.

---. (2005): “El Quijote y la novela inglesa: de Laurence Sterne a James Joyce", en Buitrago Gómez, M. C. y Senabre Sempere, R., La ficción novelesca en los Siglos de oro y la literatura europea, Madrid, Ministerio de Educación, Cultura y Deporte, pp. 57-71.

---. (2004): “El Quijote femenino como variante del mito quijotesco", en Villar Lecumberri, A., Peregrinantes peregrinos: actas del $V$ Congreso Internacional de la Asociación de Cervantistas (Lisboa 1-5 de septiembre de 2003), Vol 2, Madrid, Asociación de Cervantistas, pp. 1627-1644.

---. (1997a): La tradición cervantina en la novela inglesa del siglo XVIII. Salamanca: Universidad de Salamanca.

---. (1997b): "Formas de imitación del Quijote en la novela inglesa del siglo XVIII: Joseph Andrews y Tristam Shandy, Anales Cervantinos 33, pp. 133-164.

PAUL, A. (2009): "Feminine Transformations of the Quixote in Eighteenth-Century England", en Garrido Ardila, J.A, The Cervantean Heritage. Reception and Influence of Cervantes in Britain, Oxford, Legenda, Modern Humanities Research Association, pp. 166-175. 
Paulson, R. (1998): Don Quixote in England. The Aesthetics of Laughter, Baltimore y London, The Johns Hopkins University Press.

Raven, J. (2003): “The Anonymous Novel in Britain and Ireland, 1750-1830", en Griffin, R. J., The Faces of Anonymity: Anonymous and Pseudonymous Publications from the Sixteenth to the Twentieth Century, New York, Palgrave. pp. 141-166.

Reeve, C. (1930): The Progress of Romance and the History of Charoba, Queen of Aegypt. Reproduced from the Colchester Edition of 1785, New York, The Facsimile Text Society.

Roper, D. (1978): Reviewing before the Edinburgh 1788-1802, London, Methuen \& Co.

Seccombe, T. (1909): The Age of Johnson (1748-1798). London: George Bell and Sons.

Shaftesbury, A. A. C. (1997): Carta sobre el entusiasmo, Barcelona, Crítica.

Williams, I. (ed.), (1970): Novel and Romance 1700-1800. A Documentary Record, London: Routledge \& Kegan Paul.

Zıolkowski, E. J. (1991): The Sanctification of Don Quixote. From Hidalgo to Priest, University Park, Pennsylvania: The Pennsylvania State University Press.

Mónica Amenedo-Costa

Universidade da Coruña

mamenedo@udc.es 
